\title{
Rivaroxaban versus Aspirin in Prevention of Venous Thromboembolism: A Meta-Analysis of 9 Randomized Controlled Trials comprising 7,656 Patients
}

\author{
${ }^{1}$ Department of Cardiology, the First Affiliated Hospital of Guangxi \\ Medical University, Guangxi Cardiovascular Institute, Nanning, \\ Guangxi, China \\ 2 The first Clinical Medical College, Guangxi Medical University, \\ Nanning, Guangxi, China
}

Jian Xie ${ }^{1}$ Mingyang Jiang ${ }^{2}$ Yunni Lin ${ }^{2}$ Huachu Deng ${ }^{2} \quad$ Xiaoyong Xie $^{2}$ Lang $\mathrm{Li}^{1}$

\author{
Address for correspondence Lang Li, MD, PhD, FACC, FESC, FAICT, \\ Department of Cardiology, the First Affiliated Hospital of Guangxi \\ Medical University, Nanning, Guangxi, China \\ (e-mail: drlilang@126.com).
}

Thromb Haemost 2019;119:1517-1526.

\begin{abstract}
Keywords

- rivaroxaban

- aspirin

- venous thromboembolism

- meta-analysis.

Aim This article evaluates the preventive effects of rivaroxaban versus aspirin on venous thromboembolism (VTE) through meta-analysis of recent randomized controlled trials (RCTs).

Methods RCTs were retrieved from medical literature databases. Risk ratios (RRs) and 95\% confidence intervals ( $\mathrm{Cls}$ ) were calculated to compare the primary and safety endpoints.

Results In total, 9 trials (11 trial comparisons) were retrieved which contained 7,656 patients. Among these patients, 4,383 patients (57.2\%) received rivaroxaban, whereas 3,273 patients $(42.8 \%)$ received aspirin. Compared with aspirin, rivaroxaban significantly reduced VTE ( $1.3 \%$ vs. $3.5 \%$ ) (RR: $0.36,95 \% \mathrm{Cl}, 0.26-0.48, I^{2}=27.9 \%$ ), but significantly increased nonmajor bleeding (11.5\% vs. $7.5 \%$ ) (RR: $1.28,95 \% \mathrm{Cl}, 1.13-$ $\left.1.44, I^{2}=38.6 \%\right)$. There were no significant differences in the all-cause mortality $(0.3 \%$ vs. $0.3 \%$ ) (RR: $0.75,95 \% \mathrm{Cl}, 0.35-1.61, I^{2}=32.0 \%$ ) and major bleeding ( $0.3 \%$ vs. $0.4 \%$ ) (RR: $0.81,95 \% \mathrm{Cl}, 0.42-1.55, I^{2}=33.7 \%$ ) between the two groups.

Conclusion This meta-analysis indicated that rivaroxaban can significantly reduce the incidence of VTE when compared with aspirin. The preventive effect of rivaroxaban on VTE was more potent than that of aspirin. However, rivaroxaban had some negative side effects to patients such as nonmajor bleeding compared to aspirin.
\end{abstract}

\section{Introduction}

In recent years, the incidence of venous thromboembolism (VTE) has been on the rise. ${ }^{1}$ The sequelae of thrombosis seriously affect the functionality of patients and even lead to disability. ${ }^{2}$ Pulmonary embolism (PE) caused by undiagnosed or untreated deep vein thrombosis (DVT) contributes to a mortality rate of $70 \%$ among patients. ${ }^{3}$ Currently, the main clinical anticoagulants used include unfractionated heparin (UFH), low molecular weight heparin (LMWH), warfarin, sodium sulfate, and hirudin. However, these drugs are administered through subcutaneous or intravenous injections and are not suitable for long-term treatment. ${ }^{4}$ The traditional oral anticoagulant warfarin is difficult to control in clinical application because it interacts with many drugs and has a narrow therapeutic window. ${ }^{5}$ Therefore, rivaroxaban as a nonvitamin $\mathrm{K}$ antagonist oral anticoagulant (NOAC) which does not require routine monitoring received

March 29, 2019

accepted after revision

May 29, 2019 (c) 2019 Georg Thieme Verlag KG Stuttgart · New York
DOI https://doi.org/ 10.1055/s-0039-1693460. ISSN 0340-6245. 
of coagulation function was frequently compared with traditional anticoagulants in the past few years. ${ }^{6,7}$

Rivaroxaban is an effective direct Xa inhibitor with high oral absorption, bioavailability, and it is excreted through the kidney, bile, and feces. ${ }^{8}$ Its plasma drug concentration is dependent on the prothrombin time and activated partial thrombin time. The pharmacokinetics of rivaroxaban are dose-dependent. ${ }^{9}$ Therefore, rivaroxaban can be administered orally without the need to detect international normalized ratio (INR). Since rivaroxaban is an anticoagulant, the risk of bleeding must be carefully monitored. ${ }^{10}$

Aspirin (acetylsalicylic acid) can irreversibly inhibit cyclooxygenase and the synthesis of thromboxane A2 in platelets. It has a potent antiplatelet aggregation effect. ${ }^{11}$ Studies have shown that aspirin can prevent DVT and PE. ${ }^{12,13}$ But whether it can be used as a routine drug to prevent venous thromboembolic is controversial.

On this basis, we therefore performed this meta-analysis to compare the effects of rivaroxaban versus aspirin on VTE to provide a theoretical basis for their clinical use.

\section{Methods}

\section{Search Strategy}

Four researchers retrieved published studies comparing the efficacy of rivaroxaban versus aspirin in the prevention of VTE following the Preferred Reporting Items for Systematic Reviews and Meta-Analyses (PRISMA) guidelines. ${ }^{14}$ The randomized controlled trials (RCTs) were systematically searched in the databases such as Cochrane Library, Embase, PubMed, Baidu Scholar, Google Scholar, China National Knowledge Infrastructure, and China Science and Technology Journal Database (VIP) with no restrictions on publication date or language from inception to February 11, 2019. The following keywords and Medical Subject Headings terms were used: "5-chloro-N-(((5S)-2-oxo-3-(4-(3-oxomorpholin-4-yl)phenyl)-1,3-oxazolidin-5-yl)methyl)thiophene-2carboxamide," “Xarelto," “BAY 59-7939," "BAY 59 7939," "BAY 597939," "Acetylsalicylic Acid," "Acid, Acetylsalicylic," "2-(Acetyloxy)benzoic Acid," “Acylpyrin," "Aloxiprimum," "Colfarit," "Dispril," "Easprin," "Ecotrin," “Endosprin," “Magnecyl," “Micristin," “Polopirin," “Polopiryna," “Solprin," "Solupsan," "Zorprin," "Acetysal," "Venous Thromboembolism," and "Thromboembolism, Venous." Additional relevant studies were retrieved from meta-analyses, reviews, and other literature. Three authors screened and doublereviewed the retrieved studies. Where disputes were encountered, they were resolved by consulting a fourth author. In this meta-analysis, all data were extracted from previously published studies, thus no patient consent and ethical approval were required.

\section{Inclusion and Exclusion Criteria}

Relevant clinical trials were retrieved to evaluate the effect of rivaroxaban versus aspirin in the prevention of VTE. The inclusion criteria consisted of the following: RCTs that involved rivaroxaban and aspirin; all patients who had been diagnosed with DVT and PE according to the interna- tional diagnostic guidelines of these two conditions; RCTs that involved primary prevention of VTE associated with surgical procedures or secondary prevention of VTE recurrence; studies that allocated patients into two groups (rivaroxaban and aspirin treatment groups); and language was limited to English or Chinese. The exclusion criteria consisted of the following: nonrandomized clinical trials, case reports, series reviews, or animal experiments; retrospective trials; studies with erroneous or incomplete data; studies with rivaroxaban or aspirin allergic patients; and studies with results that were not focused on VTE (DVT or PE).

\section{Endpoints}

The primary endpoints for this study were the VTE and allcause mortality. The safety endpoints included major bleeding and nonmajor bleeding (any bleeding, clinically relevant nonmajor bleeding, minor bleeding, incision bleeding, ecchymosis, subcutaneous ecchymosis, etc.).

\section{Data Extraction}

Three authors independently reviewed the contents of the retrieved studies. The primary endpoints and safety endpoints were extracted by two authors and verified by a third author. The data extracted included the following primary information: first author's name, year of publication, country of study, sample size, average age, intervention, follow-up time, and endpoints measured in each study. If the contents of the studies needed clarification, the first author of the study was contacted. Disagreements were resolved through consensus or by consulting a third author.

\section{Risk-of-Bias Assessments}

The methodological quality of the included studies was estimated independently by two authors based on The Cochrane Risk of Bias criteria. Each quality item was graded as low risk, high risk, or no clear risk. The seven items used to assess bias in each trial included randomization sequence generation, allocation concealment, blinding of participants and personnel, blinding of outcome assessment, incomplete outcome data, selective reporting, and other biases.

\section{Statistical Analysis}

Stata (version 12.0, Stata Corp, College Station, Texas, United States) was used to analyze and pool the individual research results. Pooled results were recorded as risk ratios (RRs) and 95\% confidence intervals (CIs) with two-sided $p$-values. $p$-Values $<0.05$ were considered to be statistically significant. Heterogeneity was evaluated using the $I^{2}$ test. The heterogeneity was considered to be small when $I^{2}<50 \%$ and substantial when $I^{2}>50 \%$. The fixed effect model was used when $I^{2}<50 \%$, while the random effect model was used when $I^{2}>50 \%$. A funnel plot was generated to examine the publication bias and to explore the sources of heterogeneity. The subgroup analysis was performed according to the dosage of rivaroxaban $(10-20 \mathrm{mg}$ according to the administration dose of rivaroxaban per day), dosage of aspirin (100 $\mathrm{mg}$ and other according to the 
administration dose of aspirin per day), follow-up time (less than 1 month, 1 month, 3 months, and 12 months groups), and country of study (Canada and China).

\section{Results}

\section{Studies Retrieved and Characteristics}

A total of 13,897 relevant studies were enrolled according to the PRISMA guidelines. The titles and abstracts of the studies were screened to exclude irrelevant studies. Then, we further eliminated the unfit studies by reading the full text of the articles. Finally, 9 studies (10 trial comparisons) were included according to the inclusion and exclusion criteria and they had a total of 7,656 patients as shown in - Fig. 1. ${ }^{15-23}$ In general, 4,383 patients (57.2\%) were randomized to rivaroxaban groups, whereas 3,273 patients (42.8\%) were randomized to aspirin groups. All studies included in this metaanalysis were RCTs. The basic characteristics of the individuals from the trials are described in - Table 1.

\section{Literature Quality Evaluation}

The Cochrane Risk of Bias criteria was used to evaluate the quality of the retrieved studies by two authors. The included studies were all RCTs. Nine studies described random sequence generation and allocation concealment. ${ }^{15-23}$ Two studies described blinding of participants and personnel. ${ }^{22,23}$ Two studies described blinding of outcome assessment. None of the studies described other biases. The literature quality score is shown in - Table 2.

\section{Primary Endpoints}

\section{The Incidence of VTE}

Nine studies (11 trial comparisons) reported the incidence of VTE. ${ }^{15-23}$ In general, 58 out of 4,383 patients in the rivaroxaban groups developed VTE, whereas 116 out of 3,273 patients in the aspirin group developed VTE. This showed that rivaroxaban could significantly reduce the incidence of VTE compared to aspirin (1.3\% vs. 3.5\%) (RR: $0.36,95 \% \mathrm{CI}, 0.26-0.48, I^{2}=27.9 \%$ ) as shown in - Fig. 2 . The fixed effect model was applied to determine the level of heterogeneity among the articles, and the results showed that heterogeneity was low. The funnel plot was symmetrical indicating that there was no bias in the analysis as shown in -Supplementary Fig. $\mathbf{S 1}$ (available in the online version). Cumulative meta-analysis was performed
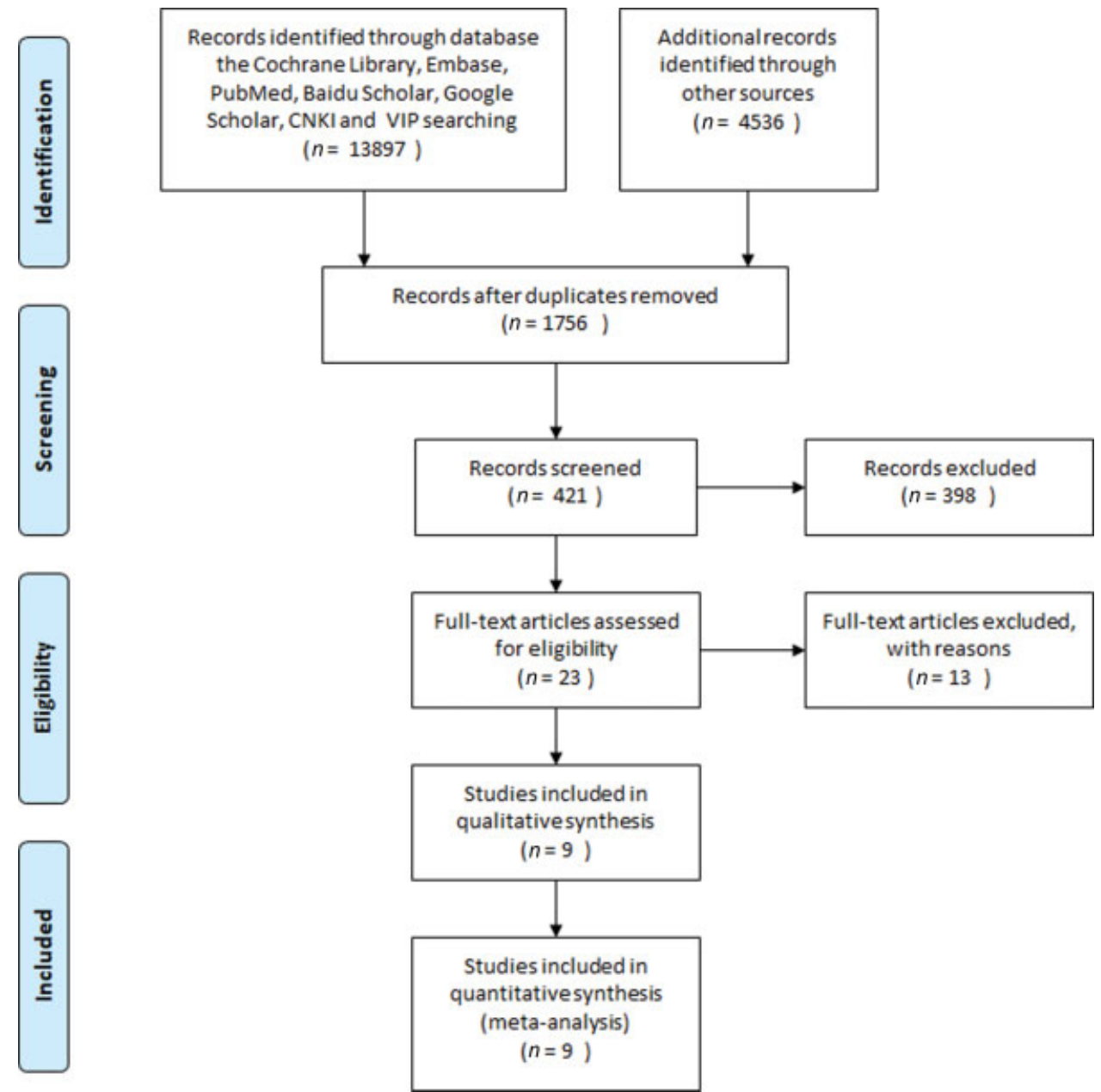

Fig. 1 Flow diagram of the study selection process. CNKI, China National Knowledge Infrastructure; VIP, China Science and Technology Journal Database. 


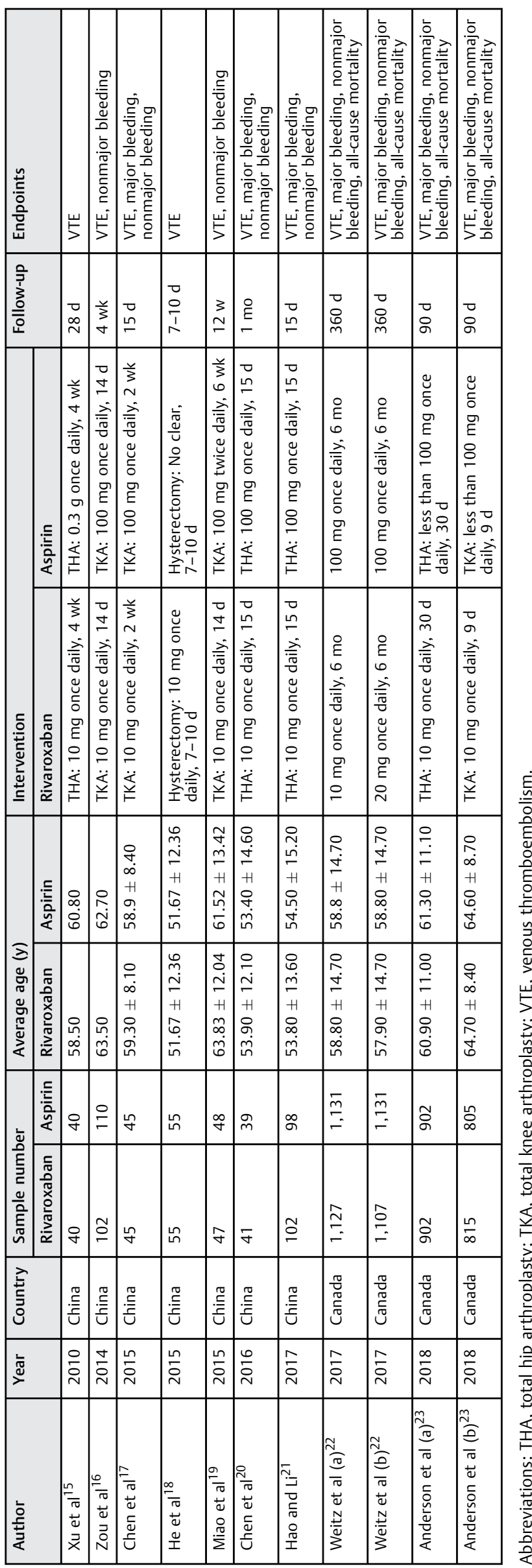
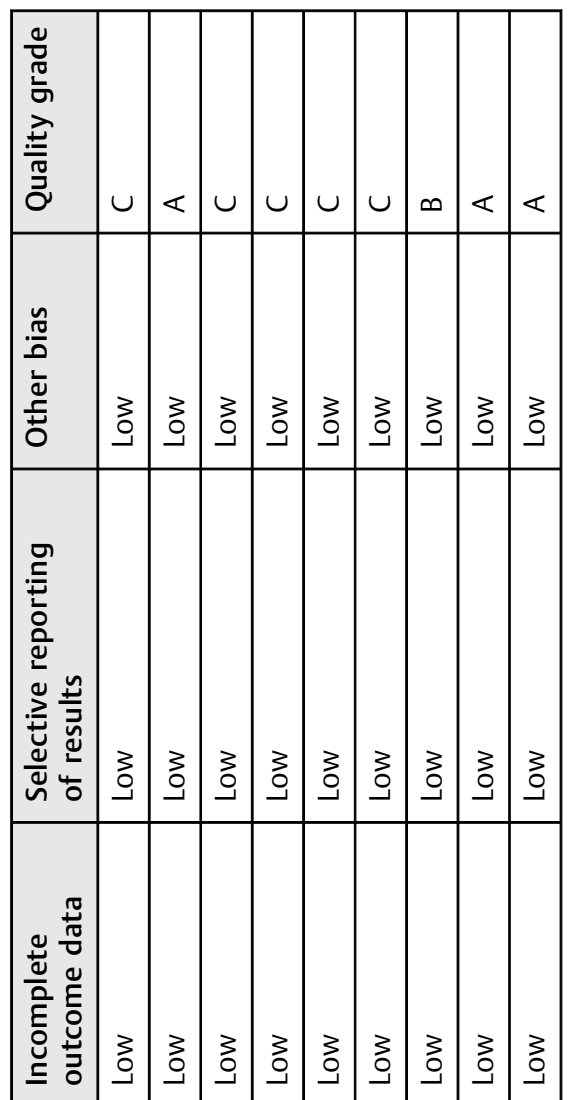

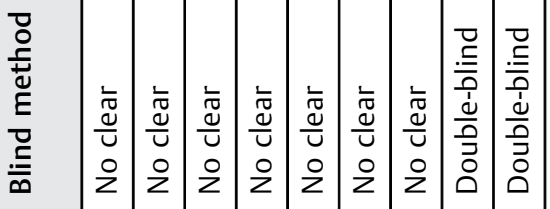
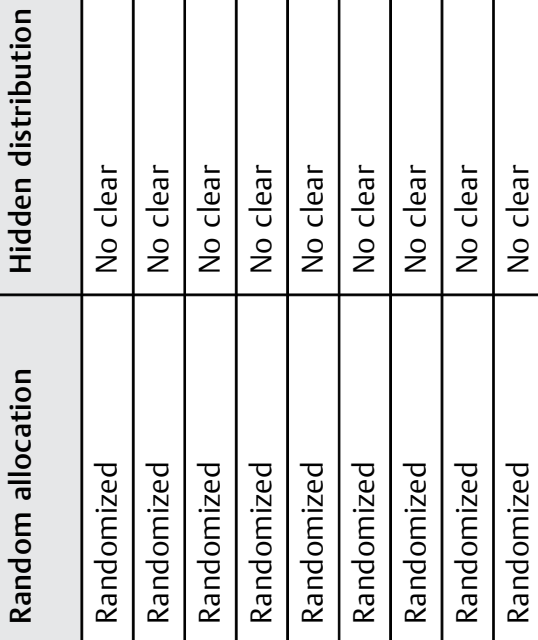


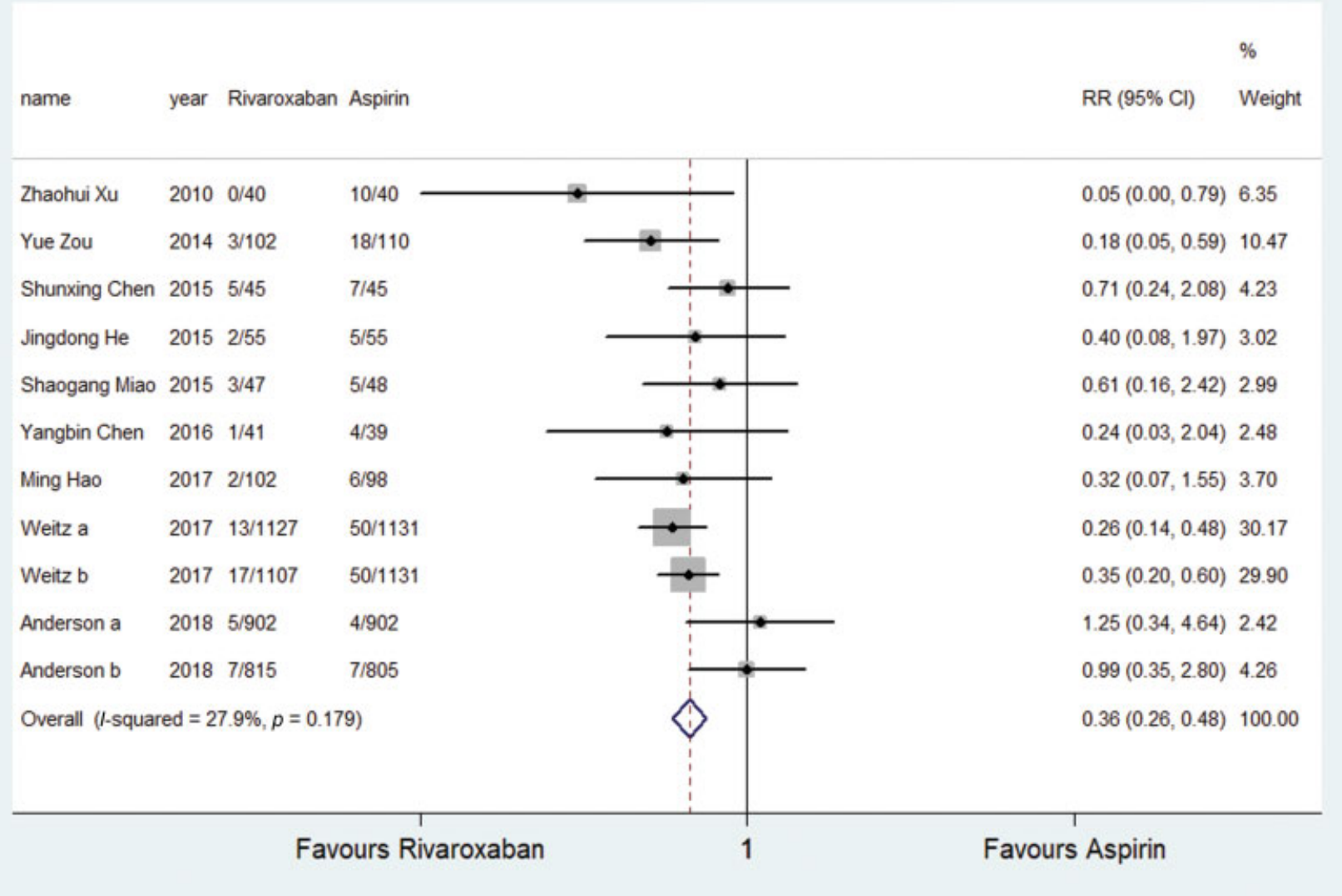

Fig. 2 Comparison of venous thromboembolism (VTE) between the rivaroxaban group and the aspirin group. RR, risk ratio.

according to the sample size from small to large, and the results showed that rivaroxaban had a better preventive effect on VTE than aspirin from the smallest sample size to the largest, and this result has been valid-as shown in -Supplementary Fig. S2 (available in the online version).

\section{Subgroup Analysis}

Subgroup analyses were performed according to the dosage of rivaroxaban, dosage of aspirin, type of surgery, medication duration, follow-up time, and country of study.

In the dosage of rivaroxaban subgroup analysis, the results showed that rivaroxaban significantly reduced the incidence of VTE at both 10 and $20 \mathrm{mg}$ dosage compared with aspirin (RR: $0.36,95 \% \mathrm{CI}, 0.25-0.51$; RR: $0.35,95 \% \mathrm{CI}$, 0.20-0.60), as shown in -Supplementary Fig. $\mathbf{S 3}$ (available in the online version).

In the dosage of aspirin subgroup analysis, it was also found that rivaroxaban significantly reduced the incidence of VTE at $100 \mathrm{mg}$ dosage compared with aspirin (RR: 0.32, 95\% CI, 0.23-0.44); there were no significant differences in the other doses between the two groups (RR: 0.54, 95\% CI, 0.291.03), as shown in -Supplementary Fig. $\mathbf{S 4}$ (available in the online version). It is likely that the differences in dosing and duration of aspirin treatment may be the cause of the poor efficacy in other groups.
In the type of surgery subgroup analysis, the results showed that rivaroxaban significantly reduced the incidence of VTE at total hip arthroplasty (THA), total knee arthroplasty (TKA), and other groups compared with aspirin (RR: 0.34 , 95\% CI, 0.16-0.74; RR: 0.50, 95\% CI, 0.29-0.86; RR: 0.31, 95\% CI, 0.21-0.46), as shown in - Supplementary Fig. S5 (available in the online version).

In the medication duration subgroup analysis, the result showed that rivaroxaban significantly reduced the incidence of VTE at 2, 4, and more than 4 weeks groups compared with aspirin (RR: 0.40, 95\% CI, 0.22-0.72; RR: 0.05, 95\% CI, 0.00-0.79; RR: 0.39, 95\% CI, 0.20-0.73); there were no significant differences at less than 2 weeks group between the two groups (RR: $0.75,95 \% \mathrm{CI}, 0.31-1.80$ ), as shown in -Supplementary Fig. $\mathbf{5 6}$ (available in the online version). The ineffective result in the less than 2 weeks group was mainly due to the insufficient duration of medication, which did not reach the lowest level of reflecting difference. In the follow-up time subgroup analysis, it was found that rivaroxaban significantly reduced the incidence of VTE at 1 and 12 months groups compared with aspirin (RR: 0.14, 95\% CI, 0.05-0.38; RR: 0.30, 95\% CI, 0.20$0.46)$; there were no significant differences at less than 1 and 3 months groups (RR: 0.49, 95\% CI, 0.23-1.06; RR: 0.94, 95\% CI, 0.47-1.88), as shown in -Supplementary Fig. 57 (available in the online version). Since the number of 
studies in different subgroups analyses was small and displayed dissimilar baseline characteristics, this study could not explain the cause of differences in the effects of the two drugs at various follow-up times.

In the country subgroup analysis, the result showed that the use of rivaroxaban significantly reduced the incidence of VTE both in the China and Canada groups compared with aspirin (RR: 0.30, 95\% CI, 0.18-0.51; RR: 0.38, 95\% CI, 0.270.54 ), as shown in -Supplementary Fig. $\mathbf{S 8}$ (available in the online version).

\section{All-Cause Mortality}

Two studies (four trial comparisons) reported all-cause mortality. ${ }^{22,23}$ A total of 11 out of 3,951 patients in the rivaroxaban group died from various causes. Eight patients out of 2,838 in the aspirin group died from various causes. These results showed that there were no significant differences in all-cause mortality between the rivaroxaban and aspirin groups (0.3\% vs. $0.3 \%$ ) (RR: $0.75,95 \% \mathrm{CI}, 0.35-$ $1.61, I^{2}=32.0 \%$ ), as shown in - Fig. 3. The fixed effect model was applied to determine the heterogeneity among the articles and the results showed that heterogeneity was low.

\section{Safety Endpoints}

\section{Major Bleeding}

Five studies (seven trial comparisons) reported major bleeding. ${ }^{16,19,21-23}$ In total, 16 out of 5,294 patients in the rivaroxaban group experienced major bleeding, while 11 out of 4,205 patients in the aspirin group experienced major bleeding. This showed that there were no significant differences in major bleeding between the rivaroxaban and aspirin groups (0.3\% vs. $0.4 \%$ ) (RR: $0.81,95 \% \mathrm{CI}, 0.4235-$ $\left.1.55, I^{2}=33.7 \%\right)$, as shown in - Fig. 4. The fixed effect model analysis revealed that heterogeneity was low among the articles.

\section{Nonmajor Bleeding}

Seven studies (nine trial comparisons) reported nonmajor bleeding. ${ }^{15,16,18,20-23}$ In total, 493 out of 4,288 patients in the rivaroxaban group experienced nonmajor bleeding, while 238 out of 3,178 patients in the aspirin group experienced nonmajor bleeding. This showed that rivaroxaban remarkably increased the incidence of nonmajor bleeding compared with aspirin (11.5\% vs. $7.5 \%$ ) (RR: $1.28,95 \%$ CI, 1.13-1.44, $I^{2}=38.6 \%$ ), as shown in - Fig. 5. Moreover, the fixed effect model revealed that heterogeneity was low among the articles.

\section{Discussion}

VTE is the most common cause of in-hospital mortality. It is associated with complications which require intense medical treatment. ${ }^{24}$ Rivaroxaban was the first direct Xa inhibitor to be recommended for clinical application. It overcomes the limitations of traditional anticoagulants in

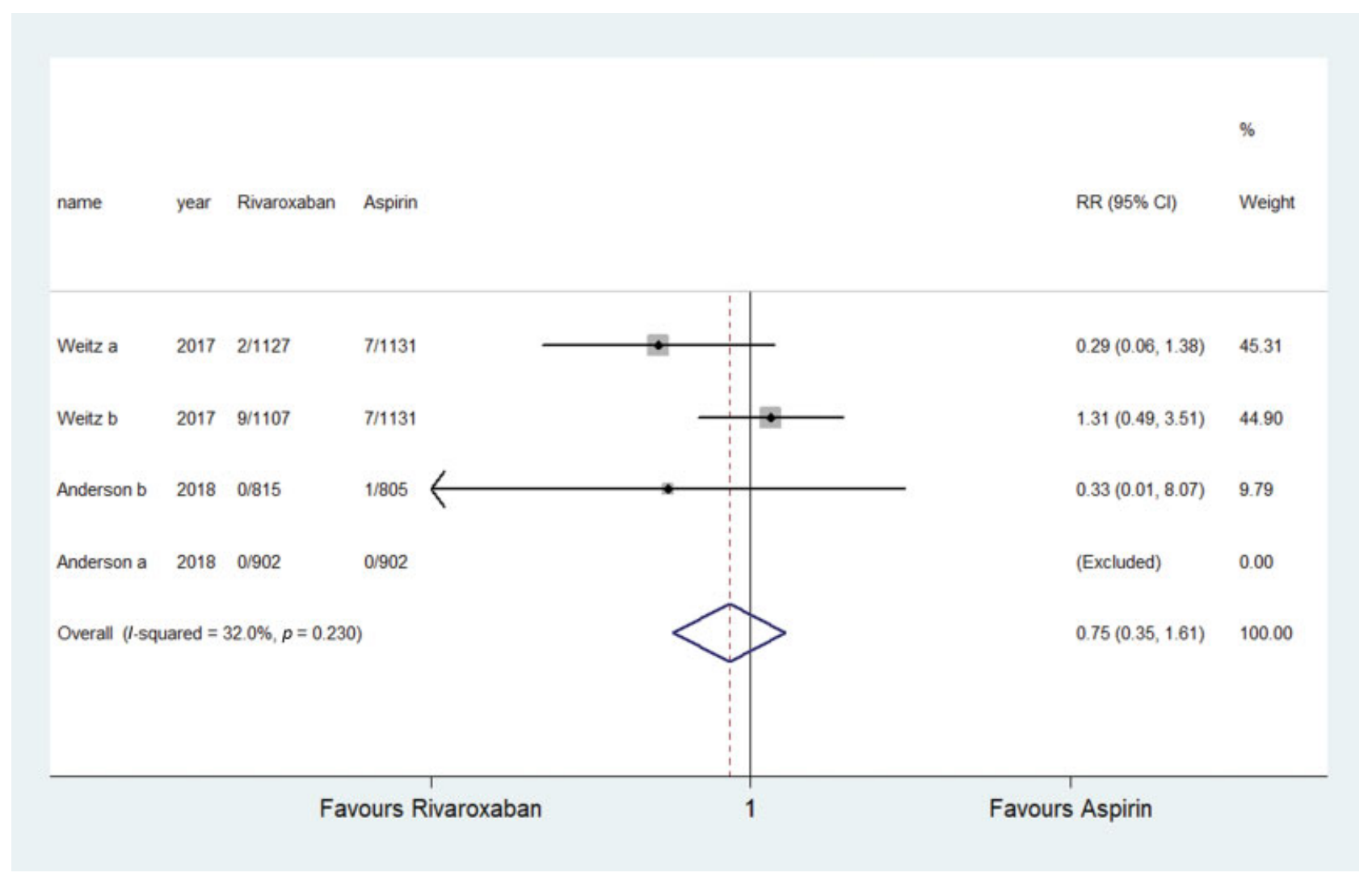

Fig. 3 Comparison of all-cause mortality between the rivaroxaban group and the aspirin group. RR, risk ratio. 


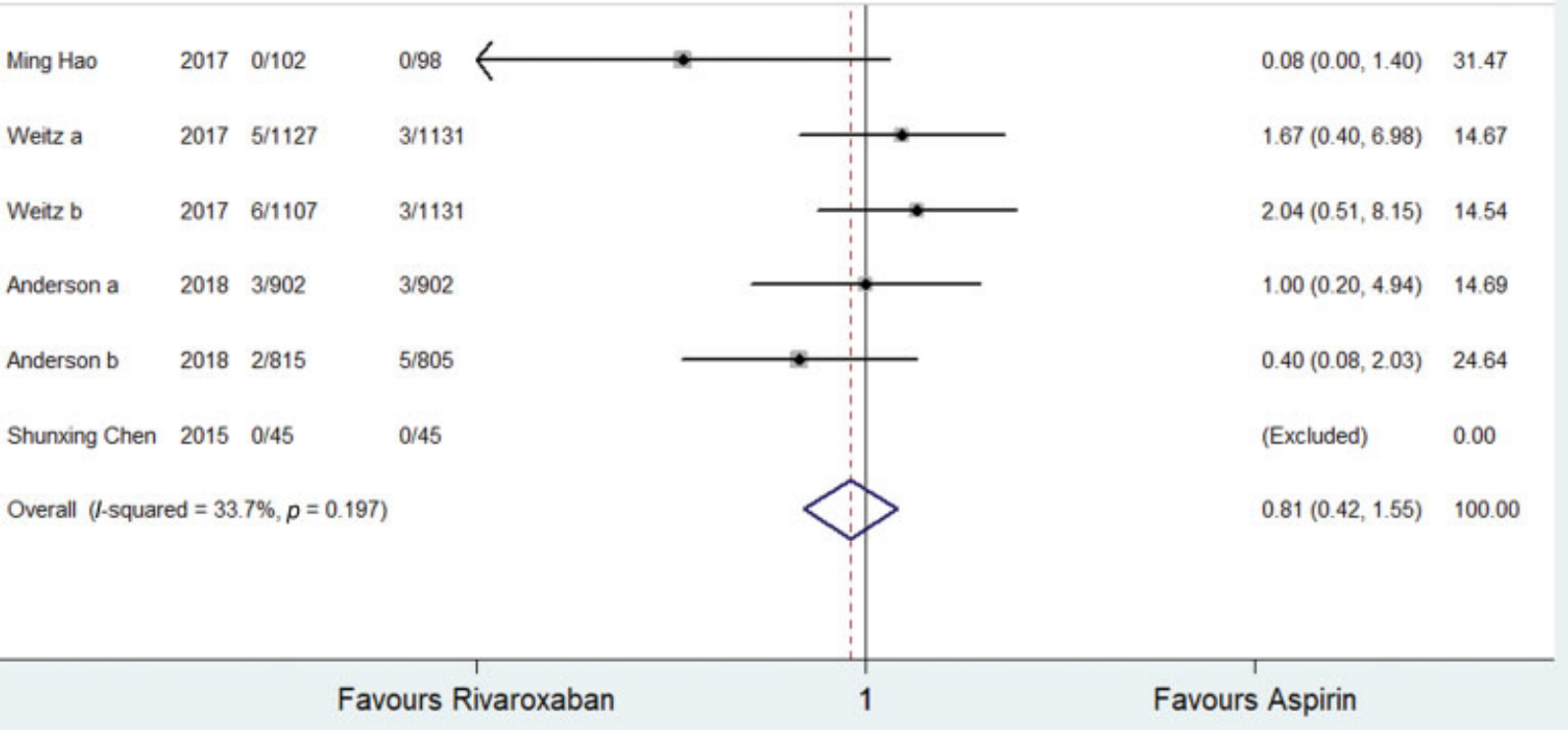

Fig. 4 Comparison of major bleeding between the rivaroxaban group and the aspirin group. RR, risk ratio.

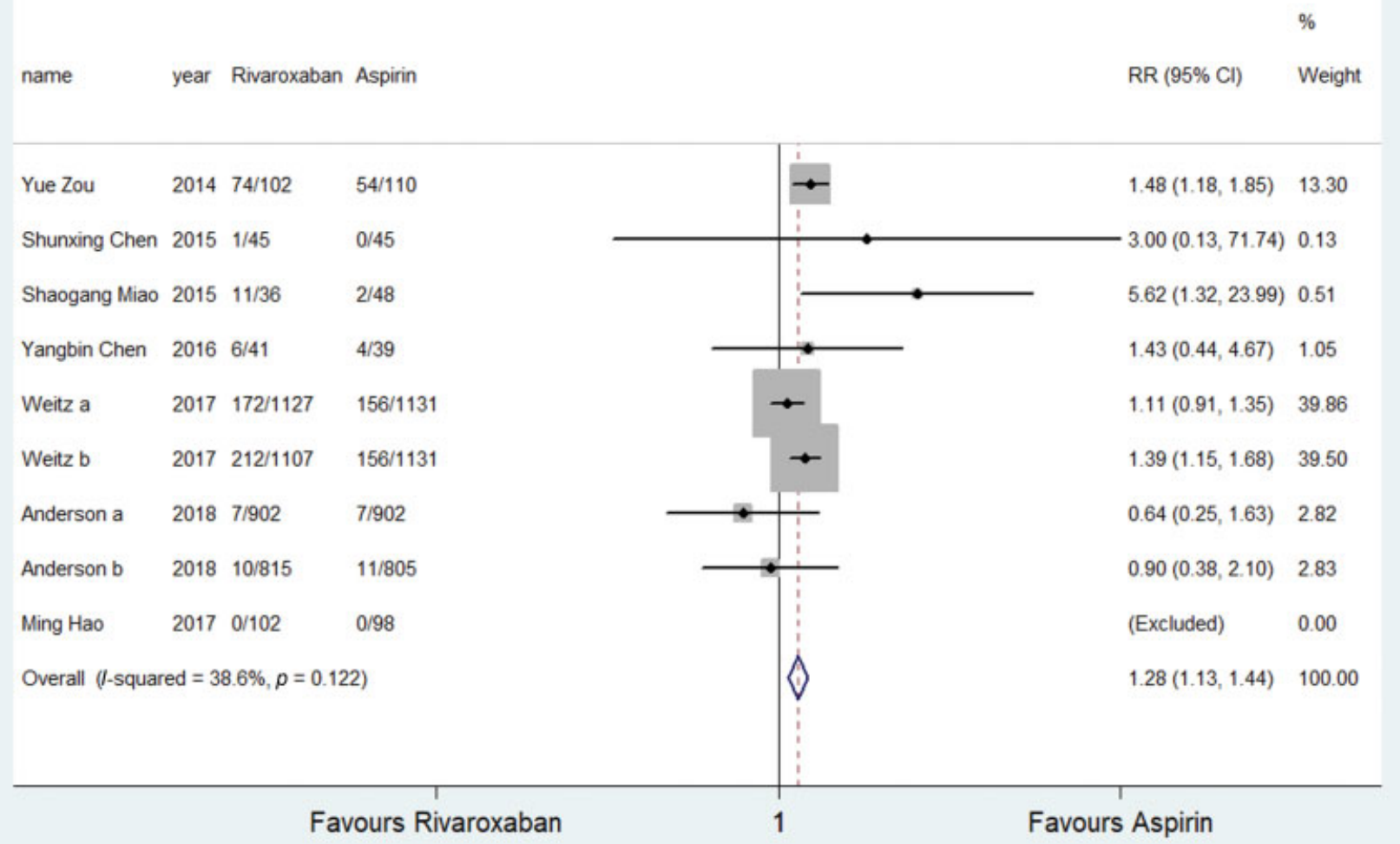

Fig. 5 Comparison of nonmajor bleeding between the rivaroxaban group and the aspirin group. RR, risk ratio. 
preventing or treating arteriovenous thromboembolic diseases. ${ }^{4}$ Some of its advantages include: convenience, rapid treatment onset, and low pharmacologic interactions with other drugs. ${ }^{9}$ This is the first meta-analysis to evaluate the effect and safety of rivaroxaban in the prevention of VTE. The results of this meta-analysis show that the incidence of VTE in the rivaroxaban group was significantly lower than that in the aspirin group (RR: $0.36,95 \% \mathrm{CI}, 0.26-0.48$, $I^{2}=27.9 \%$ ). Rivaroxaban did not increase the incidence of all-cause mortality (RR: $0.75,95 \% \mathrm{CI}, 0.35-1.61, I^{2}=32.0 \%$ ) and major bleeding (RR: 0.81, 95\% CI, 0.42-1.55, $I^{2}=33.7 \%$, but it increased the incidence of nonmajor bleeding (RR: $1.28,95 \% \mathrm{CI}, 1.13-1.44, I^{2}=38.6 \%$ ).

Constrained by economic factors, most patients are treated with traditional anticoagulants (UFH, LMWH, warfarin, sodium sulfate, hirudin, and so forth) because of economic constraints. ${ }^{6}$ However, warfarin is the only traditional oral anticoagulant approved for long-term clinical use. Some of its disadvantages are well known (frequent monitoring of coagulation, lowering of INR levels, and high mortality)..$^{5}$ Therefore, in addition to educating patients and doctors about the side effects of anticoagulants, there is a need for newer and more effective and safe NOACs. ${ }^{25}$ Currently, phase II and III clinical trials have proved that rivaroxaban can be used in THA and TKA as a NOAC. ${ }^{26}$ Its efficacy in preventing DVT after TKA is superior to that of LMWH, and it does not increase the risk of bleeding. ${ }^{27}$ The EINSTEIN series of studies (EINSTEIN-DVT study and EINSTEIN-Extension study) have shown that the efficacy of rivaroxaban in the treatment of DVT and the prevention of recurrence is comparable to the current standard treatment (enoxaparin plus warfarin).$^{28}$ The curative effect of rivaroxaban treatment for 6 to 12 months was significantly higher than that of the control group, and its safety was not different from that of the control group.

The potential clinical implications of this meta-analysis are as follows: (1) Nine RCTs ${ }^{15-23}$ were retrieved which contained a large sample size of 7,656 participants compared to previous studies. Prior studies ${ }^{29-31}$ on the prevention of VTE by rivaroxaban were limited to TKA or THA, whereas in this study, a variety of patients were investigated making the results more reliable. (2) A recent study by Intagliata et $\mathrm{al}^{32}$ reported that direct oral anticoagulants (DOACs) display similar safety characteristics when compared to traditional anticoagulants in patients with cirrhosis. They also reported that DOACs are potential attractive agents for anticoagulation therapy. As a good complement, this study is the first meta-analysis to compare the effects of rivaroxaban versus aspirin. This study also produced novel findings (the preventive effect of rivaroxaban on VTE was superior to that of aspirin), which provide a basis for the clinical application of rivaroxaban. Moreover, subgroup analyses were conducted according to the dosage of rivaroxaban, the dosage of aspirin, type of surgery, medication duration, follow-up time, and country of study to reduce the heterogeneity among the included studies and increase the reliability of these results to a certain degree. (3) In some previous studies, ${ }^{32,33}$ only the effect and safety of drugs on
DVT were analyzed, while PE was ignored. PE is a type of clinical pathophysiological syndrome triggered by pulmonary artery obstruction due to embolus shedding in the systemic circulation and its branches. This causes cerebral hypoxia and other serious consequences. ${ }^{34}$ Thus, the concept of VTE is used in our study as an endpoint, including DVT and PE. In addition, the assessment of safety endpoints was also inconsistent in previous studies. (4) Several different bleeding definitions other than major bleeding were observed among the retrieved studies (any bleeding, clinically relevant nonmajor bleeding, minor bleeding, incision bleeding, ecchymosis, subcutaneous ecchymosis, etc.). Such inconsistent definitions may affect the interpretation of the results. This study used major bleeding and nonmajor bleeding as the safety endpoints to represent the above definitions.

The limitations of this study are as follows: (1) Several baseline characteristics (diabetes, hypertension, older age, or other drug use) were not considered and this may lead to mixed bias. (2) We used the outcome events reported in the retrieved studies to integrate the results of this meta-analysis. Therefore, it is difficult to assess the effect of these baseline characteristics on the results. (3) This study could not explore the interactions among the subgroup analysis because of the limitations inherent in the included studies. (4) Though the definition of nonmajor bleeding as an endpoint was used to summarize all types of bleeding (except major bleeding) which were observed among the retrieved studies, the difference may still impact the results of our meta-analysis. (5) Only two studies described the blinding method used, which may also lead to mixed bias. (6) The two types of articles including primary prevention of VTE associated with surgical procedures and secondary prevention of VTE recurrence both met our inclusion criteria. Initial VTE patients and recurrent VTE patients were both included in some articles (such as Anderson et $\mathrm{al}^{23}$ ), but the accurate number of the two types of patients were not listed separately in these articles. Therefore, we did not know the exact number of initial VTE patients and recurrent VTE patients in each study, so we are unable to conduct subgroup analysis based on this and believe that it is reasonable to pool the data in these articles. (7) In the present analysis, most included patients came from just two studies. ${ }^{22,23}$ All the other studies are small ones (overall less than 1,000 patients), most of them published in Chinese. We conducted a cumulative meta-analysis and a subgroup about the countries (Canada and China) based on the sample size, and the results showed that the results did not change much even if the sample size was very small or only included Chinese articles. (8) The studies we included were all from China and Canada, so we also need large number RCTs of high quality and large sample sizes to confirm whether our results are applicable in other regions.

This meta-analysis reveals that rivaroxaban can significantly reduce the incidence of VTE when compared with aspirin. The preventive effect of rivaroxaban on VTE was superior to that of aspirin. However, rivaroxaban had some negative side effects such as nonmajor bleeding. 


\section{What is known about this topic?}

- It is well known that VTE (including deep-vein thrombosis or pulmonary embolism) often occurs in patients with lower extremity or pelvic or abdominal surgery, severe trauma, acute infection, pregnancy, malignant tumors, and heart disease. Therefore, for the above potential VTE population, it is necessary to carry out secondary prevention.

- Rivaroxaban, a NOAC, is an effective direct Xa inhibitor, but the application of rivaroxaban is relatively few and lacks clinical significance.

- We, therefore, performed the first meta-analysis to compare the effects of rivaroxaban versus aspirin on VTE to provide a theoretical basis for their clinical use.

\section{What does this paper add?}

- This is the first meta-analysis to compare the effects of rivaroxaban versus aspirin on VTE, making up for the gap in the efficacy and safety of NOACs and other drugs in the prevention of VTE.

- This meta-analysis reveals that rivaroxaban can significantly reduce the incidence of VTE when compared with aspirin.

- The preventive effect of rivaroxaban on VTE was more potent than that of aspirin. However, rivaroxaban had some negative side effects to patients such as nonmajor bleeding compared to aspirin.

\section{Funding}

This study was supported by the First Affiliated Hospital of Guangxi Medical University.

Conflict of Interest

None declared.

\section{Reference}

1 Naik RP, Streiff MB, Haywood C Jr, Segal JB, Lanzkron S. Venous thromboembolism incidence in the Cooperative Study of Sickle Cell Disease. J Thromb Haemost 2014;12(12):2010-2016

2 Strandness DE Jr, Langlois Y, Cramer M, Randlett A, Thiele BL. Long-term sequelae of acute venous thrombosis. JAMA 1983;250 (10):1289-1292

3 Stein PD. Pulmonary embolism following deep venous thrombosis and outcome with untreated pulmonary embolism[M] Pulmonary Embolism, Third Edition. John Wiley \& Sons, Ltd; 2016

4 Steffel J, Lüscher TF. Novel anticoagulants in clinical development: focus on factor Xa and direct thrombin inhibitors. J Cardiovasc Med (Hagerstown) 2009;10(08):616-623

5 Miranda M, Martinez LS, Franco R, Forte V, Barlattani A Jr, Bollero P. Differences between warfarin and new oral anticoagulants in dental clinical practice. Oral Implantol (Rome) 2016;9(03):151-156

6 Hum J, Shatzel JJ, Jou JH, Deloughery TG. The efficacy and safety of direct oral anticoagulants vs traditional anticoagulants in cirrhosis. Eur J Haematol 2017;98(04):393-397

7 Mueck W, Schwers S, Stampfuss J. Rivaroxaban and other novel oral anticoagulants: pharmacokinetics in healthy subjects, spe- cific patient populations and relevance of coagulation monitoring. Thromb J 2013;11(01):10

8 Gulseth MP, Michaud J, Nutescu EA. Rivaroxaban: an oral direct inhibitor of factor Xa. Am J Health Syst Pharm 2008;65(16): 1520-1529

9 Kreutz R. Pharmacokinetics and pharmacodynamics of rivaroxaban-an oral, direct factor Xa inhibitor. Curr Clin Pharmacol 2014;9(01):75-83

10 Wasserlauf G, Grandi SM, Filion KB, Eisenberg MJ. Meta-analysis of rivaroxaban and bleeding risk. Am J Cardiol 2013;112(03): 454-460

11 Patrono C. Aspirin as an antiplatelet drug. N Engl J Med 1994;330 (18):1287-1294

12 Listed N. Prevention of pulmonary embolism and deep vein thrombosis with low dose aspirin: Pulmonary Embolism Prevention (PEP) trial. Lancet 2000;355(9212):1295-1302

13 Bourne RB. Low-dose aspirin prevented deep venous thrombosis and pulmonary embolism after surgery for hip fracture. J Bone Joint Surg Am 2000;82(12):1807

14 Moher D, Liberati A, Tetalaff J, Altman DG. The PRISMA. J Integr Med 2009;7(09):889-896

$15 \mathrm{Xu} \mathrm{ZH,} \mathrm{Qu} \mathrm{ZG,} \mathrm{Fang} \mathrm{GJ.} \mathrm{Clinical} \mathrm{observation} \mathrm{of} \mathrm{rivaroxaban}$ on prevention of deep venous thrombosis after orthopedic major hip surgery [in Chinese]. China Clin Prac Med 2010;04: $151-152$

16 Zou Y, Tian S, Wang Y, Sun K. Administering aspirin, rivaroxaban and low-molecular-weight heparin to prevent deep venous thrombosis after total knee arthroplasty. Blood Coagul Fibrinolysis 2014;25(07):660-664

17 Chen SX, Luo W, Wang GH. Comparison of efficacy and safety of aspirin and rivastatin in the prevention of venous thrombosis after total knee arthroplasty [in Chinese]. J North Pharm 2015; $\mathrm{X}: 124-125$

$18 \mathrm{He} \mathrm{JD,} \mathrm{Xie} \mathrm{LF,} \mathrm{Ma} \mathrm{XB.} \mathrm{Clinical} \mathrm{analysis} \mathrm{of} \mathrm{aspirin} \mathrm{and} \mathrm{rivaroxaban} \mathrm{in}$ the prevention of venous thrombosis after operation [in Chinese]. J Math Med 2015;X:1170-1171

19 Miao SG, Zhang XG, Lu JH, Yang Y, Lu N. Case-control study on three antithrombotic agents for the prevention of venous thromboembolism after unilateral total knee arthroplasty [in Chinese]. Zhongguo Gu Shang 2015;28(10):893-896

20 Chen Y, Wang W, Zhang L, Li J, Ma S, Fu Y. Efficacy of administering aspirin and rivaroxaban to prevent deep vein thrombosis after hip arthroplasty. Xiandai Shengwu Yixue Jinzhan 2016; 16:6916-6918

21 Hao M, Li WY. Clinical control study on efficacy of aspirin and rivaroxaban on prevention of deep vein thrombosis after total knee replacement. Yaowu Pingjia Yanjiu 2017;40:824-827

22 Weitz JI, Lensing AWA, Prins $\mathrm{MH}$, et al; EINSTEIN CHOICE Investigators. Rivaroxaban or aspirin for extended treatment of venous thromboembolism. N Engl J Med 2017;376(13): 1211-1222

23 Anderson DR, Dunbar M, Murnaghan J, et al. Aspirin or rivaroxaban for VTE prophylaxis after hip or knee arthroplasty. N Engl J Med 2018;378(08):699-707

24 Posadas-Martínez ML, Vázquez FJ, Grande-Ratti MF, de Quirós FG, Giunta DH. Inhospital mortality among clinical and surgical inpatients recently diagnosed with venous thromboembolic disease. J Thromb Thrombolysis 2015;40(02):225-230

25 Yang C, Qian J, Tang X, Gao P, Hu Y. Meta-analysis on the efficacy and adverse events of aspirin plus clopidogrel versus aspirinmonotherapy in patients with ischemic stroke or transient ischemic attack [in Chinese]. Zhonghua Liu Xing Bing Xue Za Zhi 2015; 36(12):1430-1435

26 Wood RC III, Stewart DW, Slusher L, et al. Retrospective evaluation of postoperative bleeding events in patients receiving rivaroxaban after undergoing total hip and total knee arthroplasty: comparison with clinical trial data. Pharmacotherapy 2015;35 (07):663-669 
27 Kwong LM. Rivaroxaban, an oral, direct factor Xa inhibitor: a new option for thromboprophylaxis. Orthopedics 2012;35(06): e932-e938, discussion e939

28 Cohen AT, Dobromirski M. The use of rivaroxaban for short- and long-term treatment of venous thromboembolism. Thromb Haemost 2012;107(06):1035-1043

29 Ning GZ, Kan SL, Chen LX, Shangguan L, Feng SQ, Zhou Y. Rivaroxaban for thromboprophylaxis after total hip or knee arthroplasty: a meta-analysis with trial sequential analysis of randomized controlled trials. Sci Rep 2016;6:23726

30 Trkulja V, Kolundžić R. Rivaroxaban vs dabigatran for thromboprophylaxis after joint-replacement surgery: exploratory indirect comparison based on meta-analysis of pivotal clinical trials. Croat Med J 2010;51(02):113-123
31 Ricket AL, Stewart DW, Wood RC, et al. Comparison of postoperative bleeding in THA and TKA patients receiving rivaroxaban or enoxaparin. Ann Pharmacother 2016;50(04):270-275

32 Intagliata NM, Henry ZH, Maitland $\mathrm{H}$, et al. Direct oral anticoagulants in cirrhosis patients pose similar risks of bleeding when compared to traditional anticoagulation. Dig Dis Sci 2016; 61(06):1721-1727

33 Ramacciotti E, Volpiani GG, Dávila R, Resende Aguiar VC, Silveira Magella FM, Fareed J. Early recanalization of veins in patients with DVT treated with rivaroxaban: do we need to move from bedside to bench again? Intl Angiol 2018;37(01):1-3

34 Benotti JR, Ockene IS, Alpert JS, Dalen JE. The clinical profile of unresolved pulmonary embolism. Chest 1983;84(06):669678 